\title{
Assessing HIV-related outcomes among key populations accessing community-based test and start services in Nigeria
}

Project SOAR

Follow this and additional works at: https://knowledgecommons.popcouncil.org/departments_sbsr-hiv

Part of the Demography, Population, and Ecology Commons, Family, Life Course, and Society Commons, International Public Health Commons, and the Medicine and Health Commons How does access to this work benefit you? Let us know!

\section{Recommended Citation}

Project SOAR. 2017. "Assessing HIV-related outcomes among key populations accessing communitybased test and start services in Nigeria," Activity brief. Washington, DC: Population Council. 


\section{Assessing HIV-related Outcomes among Key Populations Accessing Community-based Test and Start Services in Nigeria}

Nigeria has a generalized HIV epidemic, with an adult HIV prevalence of 3.4 percent. ${ }^{1}$ However, key populations (KPs), such as men who have sex with men (MSM) and female sex workers (FSWs), have a disproportionately higher prevalence of HIV (23 percent and 14 percent respectively). ${ }^{2}$ MSM and FSWs are confronted with pervasive stigma and poor treatment by health care workers, which negatively affect their health-seeking behaviors. ${ }^{3.4}$ KPs need respectful, accessible HIV services to address their unique needs and challenges. In particular, for those KPs who test positive for HIV, effective strategies are necessary to facilitate their timely enrollment in care and treatment initiation.

Community-based, "test and start" (TnS) service delivery is a promising strategy for increasing access to HIV testing, treatment, and care for KPs. Currently in Nigeria, community-based HIV services for KPs offer varying degrees of one-stop shopping to meet these communities' comprehensive prevention, treatment, and care needs. ${ }^{i}$ Yet little is known about the costs and effectiveness of these services-evidence that is critical for guiding the investment of resources to maximize the impact of TnS for KPs.

'PEPFAR Nigeria OSS support includes (a) some single partners who provide a full range of services, as well as (b) multiple partners each providing specific or limited elements in the range of services.

Research Partners: Population Council, Avenir Health, Federal Ministry of Health, Heartland Alliance, and USAID/Nigeria

Location: Lagos and Benue states and the Federal Capital Territory (FCT)

\section{Study Duration: 2017-2019}

For more information, contact George Eluwa, Population Council, geluwa@popcouncil.org.

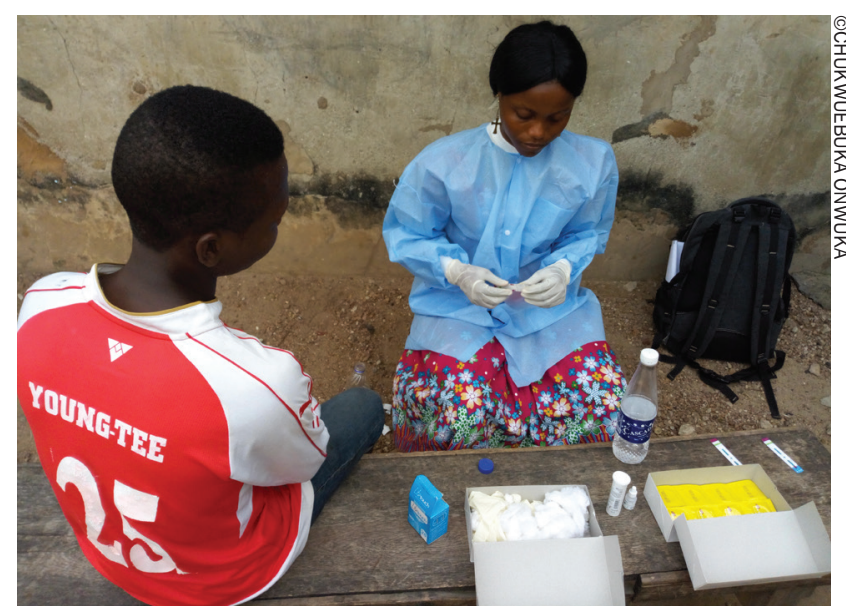

Findings from this study will be important in expanding timely ART coverage in vulnerable communities in Nigeria and elsewhere.

Project SOAR is conducting research to better understand how to use existing KP community platforms to expand access to treatment, the cost of such care provided outside of clinical health facilities, and the extent to which false positive misclassification may occur in outreach settings. Given the global "treat all" emphasis and the need to effectively scale up TnS services and minimize misclassification rates, these findings will be important in expanding timely ART coverage in vulnerable communities in Nigeria and elsewhere.

\section{Our Research}

This investigation consists of two research components: a cohort study evaluating TnS service delivery models for KPs ("TnS assessment study") and a nested study to estimate false positive misclassification rates in a subsample of these cohort participants ("misclassification study"). The overall aims are to:

- Assess and evaluate community-based TnS models in reaching KPs, providing HIV testing, linking and enrolling HIV-positive KPs in care and treatment, retaining them in ART care, and achieving viral suppression.
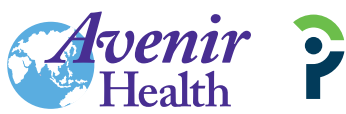

POPULATION COUNCIL Ideas. Evidence. Impact. 
- Estimate the annual operational cost per KP client for testing, linking, retaining in ART care, and achieving viral suppression.

- Assess false positive misclassification rates among a subsample of clients.

- Describe the acceptability of strategies, and optimal timing, for retesting clients to confirm diagnoses.

In the TnS assessment study, we are following for 12 months, a cohort of $600 \mathrm{KP}$ participants (FSWs and MSM) who test positive across five TnS service delivery sites: three sites in Lagos implementing a "one-stop shop" model and two sites in Benue State and the Federal Capital territory implementing a collaborative service model. ART initiation, ART retention, and behavioral outcomes of interest are being measured quantitatively at two follow-up points: 6 and 12 months. Viral load data are also being collected at 6 and 12 months.

Additionally, we are investigating factors associated with linkage, retention, and viral suppression, and assessing changes over time in a number of secondary outcomes, including HIV-related stigma, HIV disclosure, condom use, service quality, and health status. Costing analyses will estimate the operational costs of providing TnS services along primary outcomes-HIV testing, linking, retaining in ART care and achieving viral suppression.

For the misclassification study, we are recruiting a subsample of the KP cohort and selected general population participants following their initial HIVpositive rapid test result. A reference ELISA test will be utilized to identify the rate of false positive HIV test results, and a qualitative assessment will determine optimal strategies for re-testing.

\section{Research Utilization}

To ensure its relevance, we will form a Research Advisory Committee consisting of members and leaders of the MSM/FSW communities, co-investigators from local implementing partners, USAID, and government (federal and state ministries of health, National and State Agencies for the Control of AIDS). The committee will provide input into study implementation and ensure

\section{Types of KP service models in Nigeria}

One-stop shop

Currently, there are three PEPFAR-supported clinics in Lagos $^{\text {a }}$ that provide a friendly, safe-space, with each having a full-time clinical team of doctors, nurses, laboratory scientists, and pharmacists. All treatment and support services are offered on-site, and complicated cases are referred to a specialized facility.

Collaborative service model

In this model, a PEPFAR-supported partner identifies new KP clients through outreach or peer referrals, and ongoing behavior change communication and prevention services are provided. Access to ART services are facilitated by another PEPFAR partner service that specializes in ART clinical care and viral load monitoring.

${ }^{a}$ Two of these clinics are funded through USAID and the third is funded through the Military HIV Research Program/Walter Reed Program Nigeria, Department of Defense.

that the research addresses relevant evidence gaps. The committee will periodically meet during the course of the research so that members can provide input into its implementation. We will also convene a national stakeholders' meeting to share both implementation lessons and research results and to discuss their meanings and implications. The findings from the studies-both interim and final-will be reviewed with key stakeholders in light of the newly launched, 2017 revised treatment guidelines for HIV/AIDS in Nigeria.

${ }^{1}$ Federal Ministry of Health [Nigeria]. 2013. National HIV \& AIDS and Reproductive Health Survey, 2012 (NARHS Plus). Abuja, Nigera: Federal Ministry of Health.

${ }^{2}$ National HIV/AIDS \& STIs Control Programme, Federal Ministry Of Health. 2015. Nigeria Integrated Biological and Behavioural Surveillance Survey 2014. Abuja, Nigeria: Federal Ministry of Health.

${ }^{3}$ National Agency for the Control of AIDS. 2015. Federal Republic of Nigeria: Global AIDS Response Country Progress Report Nigeria. Abuja, Nigeria: National Agency for the Control of AIDS.

${ }^{4}$ Adebajo, S. B. et al. 2012. "Prevalence of internalized homophobia and HIV associated risks among men who have sex with men in Nigeria." Afr J Reprod Health. 16(4): 21-28.
Project SOAR is a five-year (September 2014-September 2019) cooperative agreement funded by the President's Emergency Plan for AIDS Relief and the U. S. Agency for International Development (Agreement No. AIDOAA-A-14-00060). SOAR is able to accept funding from all USAID accounts.

Population Council leads the Project SOAR consortium in collaboration with Avenir Health, Elizabeth Glaser Pediatric AIDS Foundation, the Johns Hopkins University, Palladium, and The University of North Carolina at Chapel Hill.
Project SOAR/Population Council

4301 Connecticut Avenue, NW, Suite 280

Washington, DC 20008

Tel: +12022379400

e-mail: ProjectSOAR@popcouncil.org

popcouncil.org/ProjectSOAR

(C)Population Council, December 2017 